\title{
Emergency Department wait time sharing: Do patients contribute to efficiency?
}

\author{
Tom R. McDougal, Jr ${ }^{1}$, Stephen J. O'Connor ${ }^{2}$, Amy Y. Landry ${ }^{2}$, Kristine R. Hearld*2 \\ ${ }^{1}$ Samford University, Department of Healthcare Administration, United States \\ ${ }^{2}$ University of Alabama at Birmingham, Department of Health Services Administration, United States
}

Received: April 4, 2017

DOI: $10.5430 /$ jha.v6n4p23
Accepted: June 18, 2017

Online Published: June 26, 2017

URL: https://doi.org/10.5430/jha.v6n4p23

\begin{abstract}
Objective: Inefficiency commonly results in overcrowding of the Emergency Department (ED) and is a problem for many hospitals. When a condition of overcrowding exists, it is often associated with lower patient satisfaction, lower care quality, and decreased financial position of the hospital. To improve patient throughput efficiency, hospitals use a variety of strategies including posting ED wait times on the hospital website and the use of ED reservation systems. This study investigates these two hospital strategies used to inform patients of anticipated wait times in the ED and their associations with patient throughput efficiency.

Methods: The study employs bivariate and Ordinary Least Squares (OLS) regression models to explore the associations between ED efficiency, measured by time spent in the ED, and ED wait times posted on the hospital website (Wait Times on Website) and ED time efficiency and the use of ED reservation systems. The sample includes all 176 acute care hospitals with an ED located in Florida.

Results: The results of this study support that posting ED wait times has a statistically significant association with time spent in the ED; however, we did not find an association between the use of a reservation system and time in the ED. Furthermore, the control variables of hospital licensed bed size, metropolitan location, percent of population without health insurance, and percent of population Medicaid eligible were found to have associations with time spent in the ED.

Conclusions: This study supports that hospitals should inform patients of anticipated ED wait times. Methods to share wait times should include posting on the hospital website, billboards, or other means to increase the likelihood of informed patients. With anticipated wait time information, the patient has the opportunity to engage in rational decision making that will positively affect ED efficiency. Healthcare leaders, including hospital administrators and ED managers, are encouraged to identify and implement better ways to inform patients of hospital performance metrics to create the opportunity for greater patient decision engagement.
\end{abstract}

Key Words: Emergency Department, Hospital performance, Wait time, Efficiency, Overcrowding

\section{INTRODUCTION}

The healthcare industry has experienced dynamic change over the past decades including pressure to improve outcomes and to be more transparent with patients and the community. Hospitals represent the largest cost centers ${ }^{[1]}$ and influencers of quality outcomes for patients ${ }^{[2]}$ and therefore have faced the greatest amount of market pressure for change and transparency of outcomes. ${ }^{[3]}$ One particular market force requiring innovation is the demand for access to Emergency Department (ED) care services. ${ }^{[4]}$

\footnotetext{
${ }^{*}$ Correspondence: Kristine R. Hearld; Email: khearld@uab.edu; Address: Department of Health Services Administration, University of Alabama at Birmingham, Birmingham, AL., 35209, United States. 
Patient demand for ED care is intensifying the current issues and causing concern for the future of ED care. From 2001 to 2006, ED visits increased by $11 \%$ while the number of EDs only increased by $4 \%{ }^{[5]}$ Additionally, there is sufficient evidence in the literature to suggest overcrowding is a growing problem for most hospitals across the country. ${ }^{[6]}$ As demand has increased for ED care, hospitals have found it necessary to improve efficiency of services and availability of resources. When resources are stretched thin, ED efficiency decreases and a situation of overcrowding occurs.

Hospitals across the country struggle to manage the pressures associated with ED efficiency and the impact of overcrowding on the organization. ${ }^{[7]}$ A study of California hospital leaders reported that $96 \%$ of respondents believed their hospital experienced overcrowding on a regular basis. ${ }^{[8]}$ Such struggles are related to situations where the volume in the ED outstrips the resource capacity to provide timely appropriate care. It is evident in the research literature examined, that ED overcrowding has a substantial negative impact on quality of care, patient satisfaction, and hospital financial health. ${ }^{[9-12]}$ There is evidence that wait delays are impacting critical condition patients, such as heart attack victims, who in 2004 waited twenty minutes compared to only eight minutes in $1997 .{ }^{[2]}$ Of similar concern, wait times to receive care in the ED for chest pain doubled from 1988 to 2008. ${ }^{[2]}$

Hospitals increasingly are held accountable for quality performance in terms of public transparency and reimbursement. ${ }^{[13]}$ As a result, hospitals have many incentives to improve ED efficiency to reduce incidents of overcrowding and the subsequent negative influences on both hospital and patient.

As a result, hospitals use many strategies to address and improve ED efficiency. Such strategies include internal efficiency gains through improved processes, communication, and staffing effectiveness. ${ }^{[12]}$ As with any organizational efficiency strategy, there is a maximum gain that can be achieved and the organization must then turn to other efficiency opportunities. In the case of ED efficiency, in recent years hospitals have turned to external strategies of informing patients of anticipated ED wait times. ${ }^{[14]}$ The goal of these external strategies is to inform the patient of anticipated wait times in the ED in hopes that patients will make a rational decision regarding if and when to visit an ED for care versus other healthcare setting alternatives. This study is designed to evaluate if such external strategies are effective in improving efficiency.

Overcrowding is generally defined as "to crowd to an uncomfortable or undesired excess". ${ }^{[15]}$ Related to hospital ED operations, there is not a clear and consistent definition of ED overcrowding as a result of poor ED efficiency in the literature. A literature review of 230 medical articles published between 1966 and 2002 found that a majority of ways overcrowding was defined were not explicit measurable definitions and that no clear consensus definition existed. ${ }^{[12]}$ While studies have defined overcrowding based on bed availability, volume per ED bed, and other approaches, the best method is to use time studies for actual ED performance in seeing patients in a timely manner upon arrival to the ED and in a timely manner on discharge to appropriate disposition. ${ }^{[6]}$ Unfortunately, such an approach is not evident in the literature and a commonly acceptable explicit definition does not exist.

Prior to 2010, hospitals were not required to report ED efficiency throughput metrics. However, beginning with the first calendar quarter of 2012 the Centers for Medicare and Medicaid Services (CMS) required hospitals to report hospital ED efficiency. This initiative was adopted from prior efforts of the National Quality Forum to measure ED quality. This data is now available on the CMS Hospital Compare website and includes the following metrics: Median Time from ED Arrival to Provider Contact for ED Patients, Median Time from ED Arrival to ED Departure for Discharged ED Patients, Median Time from ED Arrival to ED Departure for Admitted ED Patients, and Admit Decision Time to ED Departure Time. ${ }^{[16]}$ Upon review of the literature, there is no evidence that these variables have previously been used to define ED efficiency and the condition of overcrowding. However, these variables serve as a consistent measurement of ED efficiency and potential overcrowding in this study.

The possible association of sharing anticipated ED wait times with patients and the impact on efficiency improvement is a curious and unexplored relationship. The two primary strategies are to share current ED wait times or to use an ED reservation system. Both strategies are designed with a singular purpose of encouraging the patient to choose the ED wisely for care when they are informed in advance of anticipated time to receive care.

The first ED wait time strategy of this study is sharing real time anticipated ED wait times with patients. This strategy commonly utilizes four methods for communication including posting wait times on the hospital website, text messaging services, mobile applications, and billboards. Use of the hospital website is the most common of these four methods, where ED wait times are posted in real time on the homepage of the hospital website.

A second ED wait time strategy is the use of a reservation system. Two commonly used reservation systems are InQuicker and ER Express. ${ }^{[17,18]}$ In a reservation system, the patient can request a reservation time to visit the $\mathrm{ED}$, typically for 
non-emergent conditions that do not require care in the next 120 minutes. Using this method, the external service provider manages the reservation system for the hospital for a fee. The patient is normally responsible for this fee although some hospitals offer the service for free to the patient. ${ }^{[19]}$ Once the patient requests an appointment time, they are generally provided a time in the next 90 to 120 minutes. ${ }^{[17]}$ Typically, the patient is guaranteed that they will be triaged within 15 minutes of their scheduled reservation time but treatment is not usually guaranteed in a particular time frame.

The purpose of this paper is to assess the relationship between ED wait time communication strategies and ED efficiency. In recent years, hospitals have increasingly shared their ED wait times with patients as a strategy to engage patients in the decision of whether or not to visit the ED for care. However, little is known if these strategies actually improve ED efficiency. The research question for this study is: "Do ED wait time communication strategies improve patient throughput efficiency?" The study design compares hospitals that share ED wait times to those hospitals that do not share ED wait times and evaluates if there is a difference in ED efficiency as evidenced by ED efficiency metrics. The literature supports the importance of ED efficiency on multiple levels and that delays in care due to long wait times have a negative impact on patient quality outcomes. Further, the literature supports that there are relationships between improved financial performance, patient satisfaction, and ED efficiency. However, there is a gap in the literature related to the effectiveness of sharing information related to anticipated ED wait times. It is assumed that this gap exists partially due to a previous lack of available data.

The hypotheses below are used to address the research question posed above:

H1: Hospitals that post ED wait times on their website will have lower ED arrival to provider contact time.

$\mathrm{H} 2$ : Hospitals that post ED wait times on their website will have lower ED arrival to departure time for discharged ED patients.

H3: Hospitals that post ED wait times on their website will have lower ED arrival to departure time for ED patients admitted as inpatients.

H4: Hospitals that post ED wait times on their website will have lower admit decision to ED departure time for ED patients admitted as inpatients.

H5: Hospitals that use an ED reservation system will have lower ED arrival to provider contact time.
H6: Hospitals that use an ED reservation system will have lower ED arrival to departure time for discharged ED patients.

H7: Hospitals that use an ED reservation system will have lower ED arrival to departure time for ED patients admitted as inpatients.

H8: Hospitals that use an ED reservation system will have lower admit decision to ED departure time for ED patients admitted as inpatients.

\section{MAterials AND MethodS}

\subsection{Materials studied}

Data for this research partially originated from the CMS data collected from acute care hospitals. In partnership with the Hospital Quality Alliance, CMS formed the Hospital Compare initiative in 2002 to collect and report hospital performance data across multiple metrics. ${ }^{[16]}$ The Hospital Compare initiative has been expanded over the years since 2002. CMS added Inpatient Mortality in 2005, Hospital Consumer Assessment of Healthcare Providers and Systems (HCAHPS) patient experience outcomes in 2008, ED efficiency metrics in 2009, and Readmission data in 2010. ${ }^{[16]}$ It should be noted that while these metrics were added in the above years, actual reporting requirements and public availability of the data occurred in subsequent years. At the beginning of 2012, Hospital Compare data metrics for ED throughput efficiency became available to the public for all hospitals nationwide to create an opportunity for comparison of performance. These data were obtained through the CMS Hospital Compare website and is publicly available for download.

Two Hospital Compare data sets, including the Hospital Data and the ED Throughput data sets, were merged into a single data file for hospital level comparison and statistical analysis. ${ }^{[16]}$ Further, this merged data set was augmented with hospital licensed bed size data from the 2013 Florida Hospital Association (FHA) ${ }^{[20]}$ and hospital community demographic data from the 2014 Area Resource File (ARF). ${ }^{[21]}$ The FHA data was available on its website for download and the ARF is publicly available for download. Finally, hospital specific strategy data were added to the data set from the websites of the sample hospitals and the reservation system companies in the sample markets. Of the 176 sample hospitals, the authors reviewed each hospital website for evidence of real time ED wait time posting and availability of a reservation system. The data related to reservation systems was verified against publicly available information from the reservation system companies InQuicker ${ }^{[17]}$ and ER Express. ${ }^{[18]}$ 
The 176 acute care hospitals in Florida with an ED were included in the study. Veterans Administration (VA) hospitals and hospitals without EDs were excluded from the study. Freestanding EDs were not included in this study as independent organizations since CMS reports this data in the host hospital consolidated data. The State of Florida was selected for the sample for two reasons. First, this study's data revealed that $60 \%$ of Florida hospitals used one of the two wait time communication strategies of this study. Second, another study survey found that $92 \%$ of hospitals in Florida reported overcrowding. ${ }^{[4]}$

It was previously noted that some hospitals use mobile apps, texting services, or billboards to post ED wait times. It was found that hospitals using these methods also posted wait times on their websites; however, not all hospitals that posted wait times on their websites also used billboards, mobile apps, or texting services. Therefore, this study uses ED wait times posted on websites as the independent variable of interest to maintain consistency.

\subsection{Methods}

The study utilized SPSS version 22 statistical software for data analysis and testing. The study included two independent variables of interest: ED wait times posted on the hospital website and ED reservation system. No hospitals in the sample used both strategies.

Four dependent variables were used to measure ED time efficiency for each hospital: time in minutes from ED arrival to provider contact for ED patients, time in minutes from ED arrival to departure for discharged ED patients, time in minutes from ED arrival to departure for admitted ED patients, and time in minutes from admit decision to ED departure admitted patients. We consider lower time in minutes to be reflective of better ED efficiency.

The control variables of this study were divided into hospital specific variables and community specific variables. The hospital specific variables included hospital licensed bed size, hospital ownership (investor owned $=1$; not investor owned $=0$ ), and an indicator of market competiveness for each hospital measured by the Herfindahl-Hirschman Index, which is the sum of square of hospital market shares in a hospital service area (HSA) as defined by the Dartmouth Atlas. The community specific variables included the location of a hospital in a metropolitan area (metropolitan $=1$; not metropolitan $=0$ ), Health Professional Shortage Area (HPSA) status (HPSA $=1$; not HPSA $=0$ ), the percent of population less than 65 years of age uninsured, the percent of population Medicaid eligible, and the percent of population over 65 years of age.

\section{RESULTS}

Table 1 is a descriptive analysis of the study variables. Of the 176 hospitals in the sample, $80(45.5 \%)$ hospitals posted their ED wait times on the hospital website and 26 (14.8\%) hospitals used a reservation system. Combined, 106 (60.2\%) hospitals used one of the two study strategies. It is notable that no hospital used both strategies of posting ED wait times on the website and use a reservation system. The mean average bed size was 286 beds and $40.9 \%$ of the hospitals were investor owned. The vast majority of hospitals were located in metropolitan areas (88.1\%) and only $8.0 \%$ of the hospitals were in HPSA designated areas. On average, $23.5 \%$ of the population in the surrounding community of the hospital were uninsured, $16.9 \%$ were Medicaid eligible, and $20.1 \%$ were over the age of 65 .

Table 1. Hospital and community characteristics

\begin{tabular}{|c|c|}
\hline & $\mathbf{N}=\mathbf{1 7 6}$ \\
\hline \multicolumn{2}{|l|}{ ED Wait Time Strategies } \\
\hline \multicolumn{2}{|l|}{ Wait Time Posted on Website } \\
\hline - $\mathrm{Yes}(\mathrm{N} / \%)$ & $80 / 45.5 \%$ \\
\hline - $\mathrm{No}(\mathrm{N} / \%)$ & $96 / 54.4 \%$ \\
\hline \multicolumn{2}{|l|}{ Reservation System } \\
\hline - Yes $(\mathrm{N} / \%)$ & $26 / 14.8 \%$ \\
\hline - $\mathrm{No}(\mathrm{N} / \%)$ & $150 / 85.2 \%$ \\
\hline \multicolumn{2}{|l|}{ Hospital Characteristics } \\
\hline - Licensed Beds (Mean/SD) & $285.6 / 239.6$ \\
\hline \multicolumn{2}{|l|}{ Ownership } \\
\hline - Investor Owned (N/\%) & $72 / 40.9 \%$ \\
\hline - Not Investor Owned (N/\%) & $104 / 59.1 \%$ \\
\hline HHI $($ Mean/SD) & $0.231 / 0.332$ \\
\hline \multicolumn{2}{|l|}{ Community Characteristics } \\
\hline \multicolumn{2}{|l|}{ Location } \\
\hline - Metropolitan (N/\%) & $155 / 88.1 \%$ \\
\hline - Non-Metropolitan (N/\%) & $21 / 11.9 \%$ \\
\hline \multicolumn{2}{|l|}{ Health Profession Shortage Area } \\
\hline - Health Professional Shortage Area & $14 / 8.0 \%$ \\
\hline - Not Health Professional Shortage Area & $162 / 82.0 \%$ \\
\hline - $\%$ Population Uninsured (Mean/SD) & $23.5 / 4.3$ \\
\hline - \% Population Medicaid Eligible (Mean/SD) & $16.9 / 4.5$ \\
\hline - \% Population > $65(\mathrm{Mean} / S D)$ & $20.1 / 6.6 \%$ \\
\hline
\end{tabular}

Table 2 presents the correlation analysis of the community characteristics variables. We did not find a correlation greater than 0.44 among the community characteristics, indicating that there is not an issue of multicollinearity among the community characteristics.

To test whether having an ED wait time strategy is associated with time spent in the ED, ordinary least squares (OLS) multiple regression analysis was employed. The results of the multivariate regression are found in Table 3. Posting

ISSN 1927-6990 E-ISSN 1927-7008 
wait times on the hospital website was statistically associated with time between ED arrival and provider contact, time between ED arrival and departure for discharged patients, and time between the admit decision and ED departure for admitted patients. Relative to hospitals with no ED wait time strategy, hospitals that post wait times had 11 fewer minutes, on average, between ED arrival and provider contact $(\mathrm{b}=-10.75, p<.001), 46$ fewer minutes between ED arrival and ED departure for discharged patients $(b=-46.07$, $p<.001)$, and 33 fewer minutes between admit decision and ED departure for admitted patients $(b=-32.82, p<.001)$. There was not a statistically significant relationship between having a reservation system and time spent in the ER.

A number of interesting findings were observed between hospital characteristics and ED time efficiency measures. In the interest of brevity, we focused on those control variables that were statistically significant across multiple ED time measures. Interested readers are referred to Table 3 for more details. There was an association between the number of licensed beds and time spent in the ER, although the practical impact is nominal. Each one unit increase in licensed beds was associated with between 0.02 and 0.07 minutes additional time in the ER for each of the ED efficiency measures. This means for each 50 additional licensed beds, patients spend between 1 and 3.5 additional minutes in the ED, on average. Patients seen in an investor owned hospital spent 13 fewer minutes between ED arrival and provider contact $(\mathrm{b}=-12.91, p<.01)$, and 13 fewer minutes between ED arrival and departure for admitted patients, on average $(b=-13.04, p<.05)$.

Table 2. Correlation matrix of community characteristics

\begin{tabular}{llllll}
\hline & $\begin{array}{l}\text { Metropolitan } \\
\text { Area }\end{array}$ & HPSA & $\begin{array}{l}\text { \% } 65 \text { without } \\
\text { Health Insurance }\end{array}$ & $\begin{array}{l}\text { \% Population } \\
\text { Medicaid Eligible }\end{array}$ & \% Population $>65$ \\
\hline Metropolitan Area & 1.0000 & & & & \\
HPSA & 0.2805 & 1.0000 & & & \\
$\%<65$ without Health Insurance & 0.0007 & 0.0400 & 1.0000 & 1.0000 & 1.0000 \\
$\%$ Population Medicaid Eligible & -0.4314 & 0.3915 & -0.1680 & -0.4346 & \\
$\%$ Population $>65$ & 0.1568 & -0.0804 & 0.0390 & \\
\hline
\end{tabular}

Table 3. Multiple regression results $(\mathrm{N}=163)$

\begin{tabular}{|c|c|c|c|c|}
\hline & $\begin{array}{l}\text { ED Arrival to } \\
\text { Provider Contact } \\
\text { Time, ED Patients }\end{array}$ & $\begin{array}{l}\text { ED Arrival to } \\
\text { Departure Time, } \\
\text { Discharged ED Patients }\end{array}$ & $\begin{array}{l}\text { ED Arrival to } \\
\text { Departure Time, } \\
\text { Admitted ED Patients }\end{array}$ & $\begin{array}{l}\text { Admit Decision to ED } \\
\text { Departure Time, } \\
\text { Admitted patients }\end{array}$ \\
\hline & b (Std. Error) & b (Std. Error) & b (Std. Error) & b (Std. Error) \\
\hline \multicolumn{5}{|l|}{ ED Wait Time Strategy } \\
\hline - No strategy & Referent & Referent & Referent & Referent \\
\hline - Reservation system & $-2.24(3.59)$ & $-7.24(16.02)$ & $3.84(7.75)$ & $5.15(11.35)$ \\
\hline - Wait Time Posted on Website & $-10.75(2.90)^{* * *}$ & $-46.07(12.95)^{* * *}$ & $-10.66(6.26)$ & $-32.82(9.17)^{* * *}$ \\
\hline \multicolumn{5}{|l|}{ Hospital Characteristics } \\
\hline Licensed Beds & $0.02(0.01)^{* *}$ & $0.07(0.02)^{* *}$ & $0.06(0.01)^{* * *}$ & $0.04(0.02)^{*}$ \\
\hline \multicolumn{5}{|l|}{ Ownership } \\
\hline - Not investor owned & Referent & Referent & Referent & Referent \\
\hline - Investor owned & $-12.91(2.72)^{* * *}$ & $-10.93(12.12)$ & $-13.04(5.86)^{*}$ & $2.51(8.59)$ \\
\hline \multicolumn{5}{|l|}{ Metropolitan Location } \\
\hline - Not metropolitan & Referent & Referent & Referent & Referent \\
\hline - Metropolitan & $7.59(6.24)$ & $50.51(27.83)$ & $32.30(13.46)^{*}$ & $38.79(19.71)$ \\
\hline HHI Hospital Index & $0.22(5.17)$ & $26.80(23.06)$ & $3.82(11.15)$ & $13.82(16.33)$ \\
\hline \multicolumn{5}{|l|}{ Community Characteristics } \\
\hline \multicolumn{5}{|l|}{ HPSA } \\
\hline - Not HPSA & Referent & Referent & Referent & Referent \\
\hline - HPSA & $1.70(5.81)$ & $2.05(25.91)$ & $10.68(12.53)$ & $-1.79(18.35)$ \\
\hline$\%<65$ without Health Insurance & $-0.31(0.33)$ & $4.67(1.46)^{* * *}$ & $1.83(0.71)^{*}$ & $3.82(1.03)^{* * * *}$ \\
\hline$\%$ Population Medicaid Eligible & $0.74(0.38)$ & $3.35(1.68)^{*}$ & $2.21(0.81)^{* *}$ & $0.72(1.19)$ \\
\hline$\%$ Population $>65$ & $0.19(0.20)$ & $-0.35(0.90)$ & $-0.04(0.44)$ & $-0.41(0.64)$ \\
\hline $\mathrm{R} 2$ & 0.4027 & 0.3452 & 0.4087 & 0.3008 \\
\hline
\end{tabular}


Among the community characteristics, the percent of the population uninsured and the percent of Medicaid eligible members were both associated with ED time measures. On average, each one unit increase in the percent of the population uninsured was associated with around 5 additional minutes between ED arrival and departure for discharged patients $(\mathrm{b}=4.67, p<.01), 2$ additional minutes between ED arrival and departure for admitted patients $(b=1.83$, $p<.05$ ), and 4 additional minutes between the admit decision and ED departure for admitted patients $(b=3.82$, $p<.001)$. Likewise, each additional unit increase in the percentage of Medicaid eligible members in a community was associated with 3 additional minutes between ED arrival and departure for discharged patients $(b=3.35, p<.05)$, and 2 additional minutes between ED arrival and departure for admitted patients $(\mathrm{b}=2.21, p<.01)$.

\section{Discussion}

Hospitals face increasing pressure to provide high quality care while maintaining financial performance. At the same time, demand for ED services continues to increase. Existent research suggests that ED wait times are related to patient satisfaction, financial performance, and quality outcomes. ${ }^{[5,10,22]}$ However, the relationship between hospital information sharing and ED wait times has not been adequately explored. This research addresses this gap in the literature by answering the following question: "Do ED wait time communication strategies improve patient throughput efficiency?"

Study results indicate a significant difference in ED wait times between hospitals with wait time communication strategies compared to those that do not have communication strategies. This study supports the idea that hospitals should post ED wait times on the hospital website and provide the same information through other methods designed to inform patients of anticipated wait times such as text messaging and mobile apps. With anticipated wait time information, the patient has the opportunity to engage in decision making that will positively affect ED time efficiency and lead to a logical decrease in the risk of ED overcrowding.

However, the use of reservation systems should not be viewed as a strategy to improve ED time efficiency and reduce the risk of overcrowding. Rather, hospitals could consider using reservation systems as a marketing differentiation strategy when ED time efficiency has not been achieved or is not a priority. It is assumed therefore that hospitals choose to offer a reservation system for a variety of reasons. One report found that the primary objective was to increase patient satisfaction as CMS has initiated incentives for hospitals that achieve certain metrics. ${ }^{[19]}$ Further, hospitals can also use reservation systems to allow patients to wait at home rather than in the ED waiting room. ${ }^{[23]}$ Another reason to offer a reservation system is for marketing purposes. ${ }^{[24]}$ From a competitive perspective, the reservation system could be an alternative to posting actual wait times.

Based on the results of this research, there are multiple implications for management practice. During data gathering, it was noted that of 176 sample acute care hospitals in Florida, 80 hospitals posted wait times on the hospital website and 26 hospitals used a reservation system. Curiously, none of these hospitals used both strategies. This study contends that while both strategies are designed to engage patients in decision making to affect ED time efficiency, selection of a particular strategy is based on the overall strategic objectives of the hospital. Thus, hospitals do not choose both strategies. Related to the 70 hospitals that did not employ either study strategy, it is assumed that these hospitals are still relying on traditional internal efficiency strategies to meet market demand for efficiency improvement. It is logical that the remaining hospitals will adopt an external strategy when it becomes necessary to extend beyond the internal strategies.

This study also revealed a statistically significant association between the ED wait time efficiency and licensed beds, the percent of the population that is uninsured and the percent of the population that is Medicaid eligible. These findings are consistent with the propensity of uninsured and underinsured patients to use the ED as a regular source of healthcare due to perceived and actual lack of access to a regular provider. ${ }^{[6]}$ From a practical standpoint, hospital managers might opt to implement ED wait time communication strategies to reduce wait times among populations that are high utilizers of care.

Although our study is the first to relate hospital communication strategies to ED wait times, several limitations that might influence our study findings are presented. First, this study only applies to acute care hospitals located in Florida. While a larger study population of a region of the country or the entire country was considered, the necessity for study of individual hospital websites for strategies employed by those hospitals limited the opportunity to choose a larger sample size. However, it should be noted that with $60 \%$ of the hospitals in Florida using one of the two strategies, it is logical that Florida hospitals would be selected for the sample for this study.

Second, at the time of this study, there was only one complete year of CMS data available related to ED throughput metrics. As explained previously, this is due to new reporting requirements of CMS in the first publicly available data in 2012 . Therefore, the ED time variables are from a short period of available data and it is not possible to compare periods or 
study performance over more than a twelve-month period. Third, as there is only one year of CMS ED throughput data, it is not possible to measure ED time efficiency pre and post wait time communication strategy implementation by a hospital. While this is interesting potential research, such an opportunity was not an option.

Fourth, there is not a consistent and widely accepted definition of ED efficiency and overcrowding in the literature. As such, this study relies on proxies for ED efficiency in the form time spent in the ED for four CMS ED throughput metrics. While it is believed that the proxies were sufficient for this study, the use of the proxy used is not supported in the literature.

Fifth, this study did not include control variables related to ED patients' access to transportation or the internet. A patient's decision of when and if to visit an ED may be influenced by their access to transportation to visit an ED versus another care location. Related to internet access, both communication strategies of this study rely on patient access to the internet. Thus, limited patient access to the internet could influence the results of this study.

Sixth and finally, the data available and collected related to hospital strategy use had limitations. Such strategy research was conducted by one author of this study and independently verified by a non-author third party. The individual websites of hospitals were surveyed in November 2014 and it had to be assumed that hospitals were using the same strategies during the CMS ED throughput data period, which was from July 2012 through June 2013. It would have been ideal to collect the strategy data during the CMS data period but such opportunity was not possible for this study.

While this study has limitations, it is not believed that any of the limitations substantially discount or devalue the findings and conclusions.

\section{Conclusions}

The results of this study are important to hospital board members and administrators that are accountable for hospital performance related to financial, quality, and satisfaction outcomes. Further, those within hospitals that have responsibility for marketing, performance, and operations should be aware of how ED time efficiency can and will impact the entire organization. Finally, clinical staff are greatly impacted by ED overcrowding and wait times. Understanding strategies to alleviate these issues can provide a better environment of care and allow clinicians to practice to their full potential.

Study results indicate several managerial implications. First, the active employment of external communication strategies to engage patients in co-management of ED wait times can be effective. Public sharing of data on wait times appears to be the most effective strategy. Using an external communication strategy combined with other initiatives targeting internal challenges is likely the most effective way to improve ED efficiency.

Second, study findings indicate that ED wait times are longer in communities with larger numbers of uninsured and underinsured individuals. Often, these patients utilize the ED as their primary source of healthcare. Although these patient groups might bring lower rates of reimbursement, hospitals are obligated to provide treatment. Given that these patients are likely to seek care in the ED, hospital managers can employ more active strategies (e.g. communication) to help improve wait times. This will benefit patients of all payer types.

Third and finally, hospital managers might consider using such external communication strategies as an opportunity to educate patients on the appropriateness of seeking ED care. When providing data on wait times, hospitals might also use this information sharing opportunity to share wait times on affiliated primary care physician offices or urgent care centers. Reducing unnecessary ED utilization can drastically reduce costs in the hospital and increase performance system wide.

Healthcare decision makers are encouraged to find and implement better ways to inform patients of hospital performance metrics to create the opportunity for greater patient decision engagement. While this study has been focused on one element of patient engagement, industry leaders should explore how patient engagement would lead to better outcomes for hospitals and their patients. It is believed that engaged patients can and will contribute through effective decision making to help hospitals improve performance across many metrics.

\section{CONFlicts of InTEREST Disclosure}

The authors declare they have no conflict of interest. 


\section{REFERENCES}

[1] Iglehart JK. The American health care system. New England Journal of Medicine. 1999; 340(5): 403-8. PMid: 9929536. https: //doi.org/10.1056/NEJM199902043400525

[2] Shover C. Study: Emergency Department Wait Times Increase. The Harvard Crimson. 2008. Available from: http://www. thecrimson. com/article/2008/1/16/stu dy-emergency-room-wait-times-increase/\#

[3] Kassirer JP. Patients, physicians, and the Internet. Health Affairs. 2000; 19(6): 115-23. PMid: 11192394. https://doi.org/10.1 377/hlthaff.19.6.115

[4] Derlet. Overcrowding in emergency departments: increased demand and decreased capacity. Annals of Emergency Medicine. 2002; 39(4): 430-2. PMid: 11919530. https://doi.org/10.1067/mem. 2002 .122707

[5] Sun BC, Hsia RY, Weiss RE, et al. Effect of Emergency Department Crowding on Outcomes of Admitted Patients. Annals of Emergency Medicine. 2012.

[6] Richards D. Overcrowding in the nation's emergency departments: complex causes and disturbing effects. Annals of Emergency Medicine. 2000; 35(1): 63-8. https://doi.org/10.1016/S019 6-0644 (00) 70105-3

[7] Ganey P. Pulse Report 2010 Emergency Department Patient Perspectives on American Health Care. 2011. Available from: https://helpandtraining.pressganey.com/Documen ts_secure/Pulse\%20Reports/2010_ED_Pulse_Report.pdf

[8] Richards JR, Navarro ML, Derlet RW. Survey of directors of emergency departments in California on overcrowding. Western Journal of Medicine. 2000; 172(6): 385. PMid: 10854386. https: //doi.org/10.1136/ewjm.172.6.385

[9] Ranney ML, Choo EK, Wang Y, et al. Emergency department patients' preferences for technology-based behavioral interventions. Annals of Emergency Medicine. 2012. PMid: 22542311. https: //doi.org/10.1016/j. annemergmed.2012.02.026

[10] Bernstein SL, Aronsky D, Duseja R, et al. The Effect of Emergency Department Crowding on Clinically Oriented Outcomes. Academic Emergency Medicine. 2009; 16(1): 1-10. https://doi.org/10.1 $111 / \mathrm{j} .1553-2712.2008 .00295 . \mathrm{x}$

[11] Rice S. Don't Die Waiting in the ER. 2011. Available from: http://www.cnn. com/2011/HEALTH/01/13/emergency . room.ep/index.html
[12] Hwang U, Concato J. Care in the emergency department: how crowded is overcrowded? Academic Emergency Medicine. 2004; 11(10): 1097-101. PMid: 15466155. https://doi.org/10.111 $1 / j .1553-2712.2004 . t b 00686 \cdot x$

[13] Wilper AP, Woolhandler S, Lasser KE, et al. Waits to see an emergency department physician: US trends and predictors, 1997-2004. Health Affairs. 2008; 27(2): w84-w95. PMid: 18198184. https: //doi.org/10.1377/hlthaff.27.2.w84

[14] Heading to the ER? Some post wait times by text, billboard: Fox News/AP; 2010. Available from: http://www. foxnews. com/story/2010/08/24/heade d-to-er-some-post-wait-times-by-text-billboard/

[15] Dictionary.com. 2014. Available from: http://dictionary.ref erence.com/browse/overcrowding

[16] CMS. Hospital Compare: CMS. 2014 [cited 2014 June 23, 2014]. Available from: http://www.cms.gov/Medicare/Quality-I nitiatives-Patient-Assessment-Instruments/Hospita 1QualityInits/HospitalCompare.html

[17] InQuicker 2013. Available from: https://inquicker.com/

[18] ER Express 2014. Available from: https://www.erexpress .co $\mathrm{m} /$

[19] No more waiting in the ED? Hospitals introduce online reservations: The Advisory Board Company. 2012 [cited 2014]. Available from: http://www .advisory. com/daily-briefing/2012/11/29 /no-more-waiting-in-the-ed-hospitals-introduce-onl ine-reservations

[20] FHA. FHA Hospital Directory. 2014. Available from: http://www . fha.org/reports-and-resources/hospi tal-directory.aspx

[21] Area Resource File. 2014 ed. Rockford, MD: US Department of Health \& Human Services; 2014.

[22] Guttmann A, Schull MJ, Vermeulen MJ, et al. Association between waiting times and short term mortality and hospital admission af ter departure from emergency department: population based cohort study from Ontario, Canada. BMJ: British Medical Journal. 2011; 342 .

[23] More Hospitals Launch Online ED Reservation Systems To Ease Waits [Internet]. 2011. Available from: http://www.ihealthbea t.org/articles/2011/5/16/more-hospitals-launch-onl ine-ed-reservation-systems-to-ease-waits

[24] Isger. Hospitals look to online reservations to ease patient waits in busy emergency rooms: Palm Beach Post; 2011. 